\title{
Análisis de los factores asociados a la calidad de vida del paciente hipertenso
}

\author{
Cristina Nolasco Monterroso, Lucía Navas Santos, Cristina Elena Carmona Moriel, Ma Dolores López Za- \\ morano, Rafael Santamaría Olmo, Rodolfo Crespo Montero \\ Universidad de Córdoba. Unidad de Gestión Clínica de Nefrología. Hospital Universitario Reina Sofía. Córdoba. \\ España
}

\section{Resumen}

Introducción: En la Hipertensión Arterial (HTA) existen una serie de factores asociados a la misma que inciden en la Calidad de Vida Relacionada con la Salud (CVRS) de la población en general, como es el caso de la Diabetes Mellitus, el hábito tabáquico, alimentación, etc.

Objetivo: Analizar la CVRS y los factores asociados a ésta, en pacientes con HTA controlados en la Unidad de Hipertensión de la Unidad de Gestión Clínica (UGC) Nefrología del Hospital Universitario Reina Sofía (HURS) de Córdoba.

Pacientes y Métodos: Se ha realizado un estudio observacional, descriptivo y transversal, en 33 pacientes hipertensos con una edad media de 73,24 $\pm 5,55$ años; 20 hombres $(60,6 \%)$ y 13 mujeres $(39,4 \%)$ controlados en la Unida de Hipertensión de la UGC Nefrología del HURS de Córdoba. Se analizó la CVRS mediante el cuestionario de salud SF-36. Para el análisis de la dependencia se utilizó el Índice de Barthel.

Resultados: Los valores de la CVRS fueron: Salud General 43.36 \pm 24.84 , Función Física 58.18 \pm 32.71 , Rol Físico 78.98 \pm 34.51 , Rol Emocional 82.07 \pm 28.50 , Función Social 81.81 \pm 33.67 , Dolor Corporal $66.06 \pm 39.42$, Vitalidad 63.83 $\pm 33,80$, Salud Mental 70.45 \pm 29.27 . La Función Física, Salud General y Dolor Corporal fueron las dimensiones en las que puntuaron más bajo que la población de referencia.

Correspondencia:

Cristina Nolasco Monterroso

C/. Sevilla 13, 14290 Fuenteovejuna. Córdoba

E-mail: cristinanolasco.cn@gmail.com
Respecto al grado de dependencia, el $45.45 \%$ eran independientes el $51.51 \%$ presentaban dependencia leve, y el $3.03 \%$ presentaban una dependencia grave. Se encontró una correlación estadísticamente significativa entre edad y la dimensión Función Física y Vitalidad; entre peso y Rol Físico y el Índice de Masa Corporal (IMC); entre ejercicio y Función Social; entre el IMC y Rol Físico y Componente Salud Física. Los pacientes con algún grado de dependencia tenían disminuida la CVRS en comparación con los independientes.

Conclusiones: A la vista de nuestros resultados podemos afirmar que, al menos en la muestra estudiada, la disminución de la CVRS está relacionada con tener algún grado de dependencia y no con padecer HTA, siendo la Salud General, la Función Física y el Dolor Corporal las dimensiones que peor puntúan respecto a la población general. El aumento de la edad y/o el IMC se relacionan con peor CVRS.

\section{PALABRAS CLAVE}

- HIPERTENSIÓN ARTERIAL

- CALIDAD DE VIDA

- DEPENDENCIA

Analysis of factors associated with the quality of life in high blood pressure patients

\section{Abstract}

Introduction: High Blood Pressure (HBP) is associated with several factors that affect the health-related quality of life (HRQOL) of the general population, such as diabetes mellitus, smoking, feeding, etc. 
Objective: To analyze the HRQOL and associated factors in patients with controlled high blood pressure in the Hypertension Unit of Clinical Management Unit (CMU) of Nephrology, University Hospital Reina Sofía (UHRS) of Cordoba.

Patients and Methods: An observational, descriptive study was conducted in 33 hypertensive patients with a mean age of $73.24 \pm 5.55$ years; 20 men $(60.6 \%)$ and 13 women (39.4\%) monitored in the Hypertension Unit of CMU of Nephrology in UHRS of Cordoba. HRQL was analyzed by the health SF-36 questionnaire. For the analysis of dependence, Barthel Index was used.

Results: HRQL values were: General Health $43.36 \pm$ 24.84; Physical Function $58.18 \pm 32.71$; Physical Role $78.98 \pm 34.51$; Emotional Role $82.07 \pm 28.50$; Social Function $81.81 \pm 33.67$; Body Pain $66.06 \pm 39.42$; Vitality $63.83 \pm 33.80$; Mental health $70.45 \pm 29.27$. Physical function, General Health and Body Pain were the dimensions in which they scored lower than the reference population. Regarding the degree of dependence, $45.45 \%$ were independent; $51.51 \%$ mild dependence and $3.03 \%$ severe dependence. A statistically significant correlation between age and Physical Function and Vitality dimensions was found; between weight and Physical Role and Body Mass Index (BMI); between exercise and Social Function; between BMI and Physical Role and Physical Health. Patients with some degree of dependency had diminished HRQOL compared to independents.

Conclusions: According to the results, it can be stated that, at least in the studied sample, lower HRQOL is associated with some degree of dependency and not with suffering from high blood pressure; being the General Health, Physical Function and Body Pain the dimensions with worse scores compared to the general population. Increased age and/or BMI are related to lower HRQOL.

\section{KEYWORDS}

- HIGH BLOOD PRESSURE

- QUALITY OF LIFE

- DEPENDENCE

\section{Introducción}

La hipertensión arterial (HTA) es un problema de salud pública de primera magnitud, tanto por su elevada prevalencia como por el importante aumento de la morbimortalidad cardiovascular que conlleva. Se ha estimado que la HTA está relacionada con el $46,4 \%$ de las muertes por enfermedades cerebrovasculares, el $42 \%$ de las muertes coronarias y el $25,5 \%$ de las muertes totales, partiendo de la distribución de presión arterial (PA) en la población española de 35 a 64 años $^{1,2}$. Según la Organización Mundial de la Salud (OMS), aproximadamente 7,1 millones de personas en todo el mundo mueren cada año a consecuencia de la $\mathrm{HTA}^{3}$.

La Sociedad Europea de Cardiología define la HTA como una elevación crónica de la presión sanguínea en las arterias, ya sea por aumento de la sistólica (PAS), diastólica (PAD) o de ambas. Es decir, se caracteriza por mantener unos niveles elevados de presión en la sangre, por encima de las demandas metabólicas del propio organismo ${ }^{4}$. Consideramos por tanto HTA cuando la PAS es $\geq 140 \mathrm{mmHg}$ y la $P A D \geq 90 \mathrm{~mm} \mathrm{Hg}^{5}$.

En la actualidad, el tratamiento para la HTA se basa en la modificación de los estilos de vida y en el uso de fármacos antihipertensivos. A pesar de ello, diferentes estudios han señalado que el control de las cifras de PA sigue siendo inadecuado en más de la mitad de los hipertensos; y esto es debido fundamentalmente al incumplimiento terapéutico y baja adherencia al tratamiento ${ }^{6}$.

La ausencia o mal control de la PA junto con otros factores de riesgo cardiovascular contribuyen a un incremento de complicaciones cardiovasculares así como daño en otros órganos blancos (riñón y cerebro) lo que hace situar esta enfermedad en la primera causa de muerte de los pacientes que la padecen y la que genera más de 5 millones de estancias hospitalarias en el territorio español ${ }^{7}$. Por este motivo, es importante que la población hipertensa tenga conciencia de este factor de riesgo así como el beneficio del control de las cifras tensionales para tener una mejor calidad de vida ${ }^{8}$.

En la población general de España la prevalencia de HTA es aproximadamente del 35\%, llegando al $40 \%$ en edades medias y a más del $60 \%$ en los mayores de 60 años, afectando en total a unos 10 millones de españoles 9 .

Los factores de riesgo asociados a la HTA se pueden resumir en: edad, género, etnia y herencia (no modificables), tabaquismo, alcohol, sedentarismo, alimentación, sociológicos y sociales (modificables comportamentales) y sobrepeso, obesidad, dislipemias, diabetes mellitus (modificables metabólicos) ${ }^{10}$. 
Los factores de riesgo no modificables o no prevenibles son inherentes al individuo; pero sin embargo, los modificables se asocian con el estilo de vida, por lo que pueden ser prevenidos, minimizados o mejor aún, eliminados ${ }^{11}$.

Estamos por tanto, ante una patología bien conocida, con un tratamiento establecido y con unos factores de riesgo modificables en parte. Aunque hay que tener presente que se trata de una patología crónica con todo lo que esto conlleva. La prevalencia de enfermedades crónicas, en la mayoría de los países, para las cuales no existe una curación total y donde el objetivo del tratamiento es eliminar los síntomas, evitar complicaciones y mejorar de este modo el bienestar de los enfermos, lleva a que las medidas clásicas de resultados en medicina como son: mortalidad, morbilidad y expectativa de vida, no sean las suficientes para evaluar la calidad de los servicios de salud surgiendo así el interés por el estudio de la calidad de vida ${ }^{12}$.

Se debe resaltar que "calidad de vida" no es lo mismo que estado de salud, estilo de vida, satisfacción con la misma, estado mental o bienestar, sino que es un concepto multidimensional que incluye la percepción que tiene el individuo sobre éstos y otros aspectos de su vida ${ }^{13}$. De este concepto, ha derivado el de Calidad de Vida Relacionada con la Salud (CVRS). La CVRS se refiere a la satisfacción del individuo en cuanto a los aspectos físicos, sociales y psicológicos de su vida en la medida en que éstos afectan o se ven afectados por su salud ${ }^{14,15}$.

Debido a que la HTA es una enfermedad poco sintomática (excepto en fases complicadas), parece difícil asociar a ella un deterioro en la CVRS. Sin embargo, hay diversos estudios y autores que afirman que la HTA produce un deterioro de la CVRS siendo ésta inferior en los pacientes hipertensos en comparación con los normotensos y dentro de los hipertensos ésta suele ser peor en el caso de aquellos que presentan comorbilidad. Ademir et al. comunicaron que los pacientes hipertensos tienen, según la evaluación del cuestionario de salud SF-36, una significativa disminución en todos los dominios de la CVRS en comparación con los pacientes normotensos ${ }^{16-18}$.

Dado a que en la HTA existen una serie de factores asociados a la misma y que estos mismos factores inciden en la CVRS de la población general, como es el caso de la Diabetes Mellitus ${ }^{19}$ o el hábito tabáqui$\mathrm{Co}^{20}$, parece interesante el estudio de la de la calidad de vida en el paciente hipertenso y los factores aso- ciados a ésta, pues habitualmente se piensa que esta patología no es limitante y este factor no está muy analizado.

Por todo ello, el objetivo de este estudio fue analizar la CVRS y los factores asociados a ésta, en pacientes con HTA controlados en la Unidad de Hipertensión de la UGC Nefrología del HURS de Córdoba.

\section{Pacientes y Métodos}

\section{Ámbito del estudio}

Este estudio ha sido realizado dentro del ámbito hospitalario, en la Unidad de Hipertensión de la UGC Nefrología, perteneciente a las Consultas Externas del HURS de Córdoba, previa autorización del director de la UGC y con la colaboración del facultativo responsable de la unidad de hipertensión y la enfermera de la misma.

\section{Población y muestra}

La población de estudio fueron pacientes diagnosticados de HTA que se controlan en la Unidad de Hipertensión de la UGC Nefrología. Se realizó un muestreo no probabilístico accidental incluyendo a todos los pacientes que acudieron a revisión en un periodo de 3 meses. Se incluyeron un total de 33 pacientes, con una edad media de 73,24 $\pm 5,55$ años.

\section{Criterios de inclusión}

Los criterios de inclusión para la participación en el estudio fueron: pacientes de ambos sexos mayores de 65 años, que hubieran aceptado participar en la investigación tras leer la hoja de información y el consentimiento informado.

\section{Diseño}

Se realizó un estudio observacional, descriptivo y transversal.

\section{Variables del estudio}

Las variables demográficas han sido: edad, sexo, peso, talla, IMC, perímetro abdominal, estado civil, ejercicio físico, hábito tabáquico, responsable en la elaboración de comidas, residencia, actividad realizada en tiempo libre, PA y frecuencia cardiaca (FC). Estas variables se obtuvieron a través de una entrevista personal, sin tener que acceder a la historia clínica del paciente.

Las variables de resultado fueron: calidad de vida, dependencia. 


\section{Instrumentos de medida}

Para determinar la calidad de vida se ha utilizado el cuestionario SF-36. Los 36 ítems del instrumento cubren las siguientes escalas: Función física, Rol físico, Dolor corporal, Salud general, Vitalidad, Función social, Rol emocional y Salud mental. Adicionalmente, el SF-36 incluye un ítem de transición que pregunta sobre el cambio en el estado de salud general con respecto al año anterior ${ }^{21}$.

Para determinar el grado de dependencia se utilizó el Índice de Barthel. El rango global de este índice, puede variar entre 0 (completamente dependiente) y 100 puntos (completamente independiente) ${ }^{22}$.

\section{Recogida de los datos}

Los datos se obtuvieron en la consulta de HTA, cuando los pacientes asistieron a revisión. En esta revisión, los pacientes estuvieron en consulta, el tiempo suficiente para contestar a las preguntas que contienen ambos cuestionarios, por lo cual no fue necesario añadir tiempo extra para la cumplimentación de los mismos. Se explicó el objetivo del estudio a cada uno de los pacientes y se les pidió firmar el consentimiento informado.

\section{Aspectos éticos}

El estudio contó con la aprobación del Comité de Ética e Investigación del HURS. La información obtenida fue confidencial y tratada conforme a la Ley Orgánica de Datos de Carácter Personal 15/99. Si en algún momento el paciente deseó retirarse del estudio lo pudo hacer sin tener que dar ningún tipo de explicación.

\section{Análisis estadístico}

Para las variables cualitativas se utilizó la distribución de frecuencias, mientras que para las variables numéricas se utilizó la media \pm la desviación estándar de la media. Para la relación entre variables se utilizó el coeficiente de Correlación de Pearson o el Test Spearman según los datos sigan o no una distribución normal. Para la comparación de grupos se utilizó la U de Mann-Whitney. Se aceptó significación estadística para $p<0.05$. Los datos se analizaron con el paquete estadístico SPSS 18.0 para Windows.

\section{Resultados}

En la Tabla I se recogen las frecuencias de las variables sociodemográficas estudiadas.
Tabla I. Variables sociodemográficas.

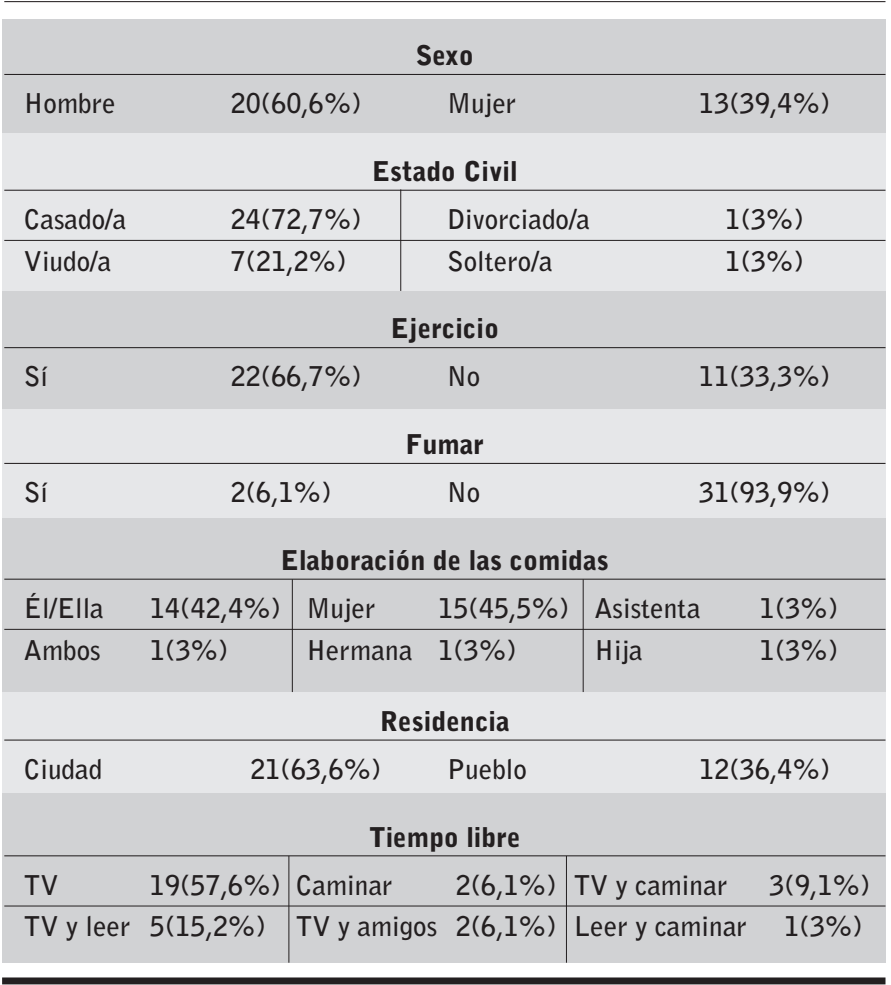

Los valores obtenidos para el resto de variables son: peso $81,02 \pm 14,77$, talla $1,64 \pm 0,08$, perímetro abdominal $107,18 \pm 13,08$, PAS $147,55 \pm 17,10$, PAD 82,18 $\pm 12,89$ y FC $69,39 \pm 11,54$.

En la Figura 1 se representan la distribución de las categorías en que se clasifica el Índice de Barthel junto con la frecuencia y porcentaje de cada grado.

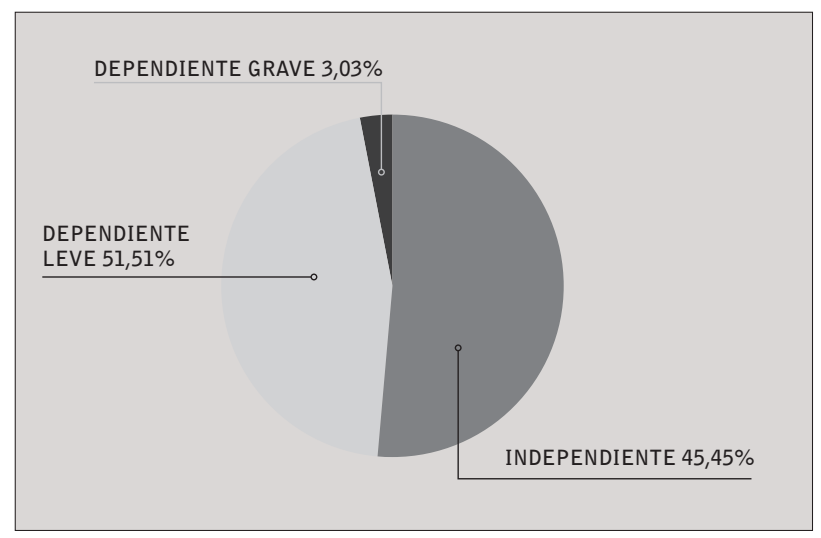

Figura 1. Categorización del Índice de Barthel.

Con respecto a la CVRS, las puntuaciones de las diferentes dimensiones se muestran en la Tabla II. 
Tabla II. Dimensiones SF-36.

\begin{tabular}{|c|c|c|c|c|c|c|}
\hline & Salud General & & Función Física & Rol Físico & Rol Emocional & Función Social \\
\hline Media & 43,36 & & 58,18 & 78,98 & 82,07 & 81,81 \\
\hline \multirow[t]{2}{*}{ Desviación típica } & 24,84 & & 32,71 & 34,51 & 28,50 & 33,67 \\
\hline & Dolor Corporal & Vitalidad & Salud Mental & Estado de Transición & Componente Salud Física & Componente Salud Mental \\
\hline Media & 66,06 & 63,83 & 70,45 & 52,27 & 62,15 & 74,54 \\
\hline Desviación típica & 39,42 & 33,80 & 29,27 & 19,13 & 25,37 & 25,68 \\
\hline
\end{tabular}

En la Figura 2 se comparan los valores de las dimensiones del SF-36 de la muestra estudiada con la población general.

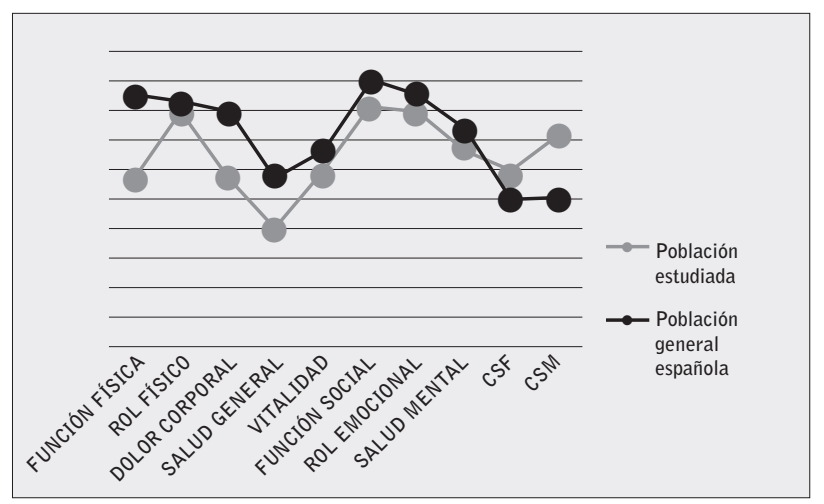

Figura 2. Valores medios de las diferentes dimensiones de la población estudiada y población general española.

Como puede apreciarse las dimensiones que más se diferenciaban con las de la población general española fueron: Función Física, Dolor Corporal, Salud General y Componente de Salud Mental (CSM) (Figura 2).

Cuando se compararon las dimensiones del SF-36 para el género, no se encontraron diferencias estadísticamente significativas. Sin embargo, cuando se comparó el sexo para el grado de dependencia, las mujeres obtuvieron una mediana de 100 (80-100) y los hombres una mediana de 85(30-100); no siendo estas diferencias estadísticamente significativas.

Para analizar el grado de dependencia respecto al resto de variables, agrupamos a los pacientes en dos categorías, teniendo en cuenta las puntuaciones del Índice de Barthel (independientes=100, algún grado de dependencia<99).

Las comparaciones para todas las dimensiones de la CVRS entre estos dos grupos se pueden observar en la Tabla III.

Tabla III. Comparación entre grupos de pacientes independientes y con algún grado de dependencia.

\begin{tabular}{l|c|c|c|}
\hline & \multicolumn{2}{|c}{ Independiente } & Algún grado de Dependencia \\
\hline Salud General & $51.14 \pm 18.13$ & $31.70 \pm 23.57$ & 0.034 \\
\hline Función Física & $73.64 \pm 27.85$ & $43.21 \pm 33.03$ & 0.023 \\
\hline Rol Físico & $100(62.50-100)$ & $84.37(0-100)$ & 0.041 \\
\hline Rol Emocional & $100(41.7-100)$ & $62.50(25-100)$ & 0.011 \\
\hline Función Social & $100(87.50-100)$ & $87.50(0-100)$ & 0.011 \\
\hline Dolor Corporal & $100(12.50-100)$ & $22.50(0-100)$ & 0.029 \\
\hline Vitalidad & $72.27 \pm 22.75$ & $44.20 \pm 36.23$ & 0.015 \\
\hline Salud Mental & $85.91 \pm 12.41$ & $52.86 \pm 31.67$ & 0.003 \\
\hline Componente de Salud Física & $74.83 \pm 16.77$ & $45.58 \pm 23.55$ & 0.002 \\
\hline Componente de Salud Mental & $89.18 \pm 10.18$ & $58.04 \pm 27.15$ & 0.002 \\
\hline
\end{tabular}


Cuando se relacionaron las distintas variables, se encontró una correlación estadísticamente significativa entre edad y la dimensión Función Física $(r=-0.631$, $p=0.01$ ); entre la edad y la dimensión Vitalidad ( $r=-$ $0.578, p=0.003)$; entre el peso y la dimensión Rol Físico ( $r=-0.506, p=0.01)$; entre el ejercicio y la dimensión Función Social ( $r=-0.446, p=0.025)$; entre el IMC y la dimensión Rol Físico ( $r=-0.518, p=0.008$ ), entre el IMC y la dimensión Componente Salud Física ( $r=$ -0.405; $p=0.044)$ y entre el peso y el IMC ( $r=0.876$; $\mathrm{p}=0.000$ ).

No se encontraron otras relaciones estadísticamente significativas.

\section{Discusión}

El objetivo del presente estudio fue analizar la CVRS y los factores asociados a ésta, en pacientes con HTA. En la actualidad, la CVRS se considera un marcador clínico más en las enfermedades de tipo crónico ${ }^{23}$, como es el caso de la HTA, pues el paciente ha de aprender a convivir con ciertas restricciones dietéticas y un tratamiento farmacológico, que pueden modificar en mayor o menor medida su estilo de vida ${ }^{24}$.

El estudio de la CVRS en el paciente hipertenso se viene realizando desde hace años, aunque no existen resultados concluyentes sobre cómo afecta la HTA a esta variable. Los diferentes estudios revisados muestran que en las dimensiones analizadas, la afectación de la CVRS tiene más que ver con las propias características de los individuos que con la propia hipertensión, como son la edad, sexo, estado civil, hábitos de vida, etc ${ }^{25}$. Otro aspecto a resaltar es que no se ha utilizado un único instrumento de medida para evaluar la CVRS en el paciente hipertenso, por lo que se han utilizado instrumentos genéricos tales como el SF-36 pues ha demostrado ser válido y fiable para las distintas poblaciones ${ }^{21}$, motivo por el cual decidimos utilizarlo en nuestro estudio.

Nuestros resultados ponen de manifiesto una peor CVRS en pacientes con HTA en algunas de las dimensiones del SF-36, como son el caso de la Función Física, Dolor Corporal y Salud General (la más afectada), cuando se compara con la población general. Estos resultados concuerdan con lo publicado por Cavalcante et al, en el que el aspecto físico es el que presenta la media más baja de todos los dominios ${ }^{25}$.
En nuestro grupo no hemos encontrado diferencias significativas entre hombres y mujeres en ninguna de las dimensiones del SF-36. Tal vez porque la muestra es pequeña y las mujeres estudiadas fueron solo 11 . Este resultado no es coincidente con varios de los estudios publicados en los cuales el sexo femenino mostró peores puntuaciones en la CVRS, en lo referente al estado de ánimo, posiblemente porque las mujeres tienen una mayor incidencia de quejas o percepción de frustración, lo que obviamente puede influir de forma global en la CVRS $^{6,26}$.

Otro factor relacionado con la CVRS es la edad. En nuestros pacientes, igual que ocurre en otra serie publicada ${ }^{4}$, existe una relación estadísticamente significativa entre la edad y las dimensiones Función Física y Vitalidad. Esto es lógico puesto que la edad hace que se acentúen las carencias de cada persona, de manera que en muchos casos se necesita ayuda de terceras personas sobre todo en el ámbito físico.

Algunos autores señalan que la CVRS en el paciente hipertenso, tiene más relación con el grado de control del tratamiento conseguido y no con la mayor 0 menor utilización de fármacos ${ }^{27}$. Sin embargo, esto en nuestro estudio no se ha podido comprobar, pues no se incluyó el tratamiento farmacológico entre las variables estudiadas.

Referente al grado de dependencia, en el grupo de pacientes de nuestro estudio, hemos visto que: el $50 \%$ presentan una dependencia leve, el $46 \%$ son independientes y sólo un $4 \%$ muestra dependencia grave. Al igual que en la serie de M. Barrantes-Monge et al, en nuestro grupo de pacientes la actividad básica más afectada fue la incontinencia urinaria seguido de la incapacidad para vestirse ${ }^{28}$. Cuando agrupamos a los pacientes según fueran o no dependientes encontramos diferencias estadísticamente significativas en todas las dimensiones del SF-36, es decir los que tenían algún grado de dependencia presentaron peor CVRS, sugiriendo este resultado que la calidad de vida está relacionada con los mismo factores que la población general sana y no con ser hipertenso. Por tanto, parece evidente que, al menos en la muestra estudiada, lo que más influye en la CVRS es el nivel de dependencia, igual que ocurre en la población general ${ }^{29,30}$.

Por otra parte, debemos decir que la evaluación de la CVRS puede ser útil para mejorar la comunicación entre pacientes y profesionales sanitarios, de manera que el objetivo asistencial contemple un cuidado integral del paciente con HTA en el que esté incluida 
la valoración periódica de la calidad de vida, como una variable más dentro del control y seguimiento de estos pacientes, que no debe limitarse sólo a reducir la PA sino también a mejorar la CVRS de los mismos, minimizando al mismo tiempo los efectos secundarios del tratamiento. Esto puede ser valioso en la ayuda global de estos pacientes ${ }^{13,23}$.

La principal limitación de este estudio es el pequeño tamaño muestral y por tanto la imposibilidad de establecer estratificaciones con grupos más amplios. Otra limitación a la hora de establecer factores asociados a la CVRS ha sido no analizar la medicación que tomaba cada paciente, porque desde la dirección de este trabajo se decidió no entrar en el tratamiento médico de los pacientes.

A la vista de nuestros resultados podemos afirmar que al menos en la muestra estudiada, la disminución de la CVRS está relacionada con tener algún grado de dependencia y no con padecer HTA, siendo la Salud General, la Función Física y el Dolor Corporal, las dimensiones que peor puntúan respecto a la población general. El aumento de la edad, el peso y/o el IMC se relacionan con peor CVRS.

Por tanto, estos resultados sugieren que los factores como la edad, el peso o la dependencia que se relacionan con la CVRS en la población general, son también los responsables del valor de esta en los pacientes hipertensos y no la HTA como entidad aislada.

Recibido: 8 agosto 2015

Revisado: 14 agosto 2015

Modificado: 20 septiembre 2015

Aceptado: 22 septiembre 2015

\section{Bibliografía}

1. Zurera I. Caballero MT. y Ruíz M. Análisis de los factores que determinan la adherencia terapéutica del paciente hipertenso. Enfermería Nefrológica; 2014; 17(4):251-260.

2. Fontenla A. García JA. Hernández F. Y cols. Manejo de la hipertensión resistente en una unidad multidisciplinaria de denervación renal: protocolo y resultados. Revista Española de Cardiología; 2013; 66(5) 364-370.

3. Muñoz M. Autocuidado en el adulto hipertenso. Tesis. 2013.

4. Vinaccia S. Quiceno M. Gómez A. Montoya M. Calidad de vida relacionada con la salud en pacientes con diagnóstico de hipertensión arterial leve. Diversitas; 2007; 3(2).

5. Mancia G. Fagart R. Narkiewicz K. y cols. Guía de práctica clínica de la ESH/ESC para el manejo de la hipertensión arterial. Hipertensión y riesgo vascular; 2013; 30(3):1.

6. Gil VF. Esparcia y Merino J. Calidad de vida en el hipertenso y factores que la determinan. Hipertensión; 2001; 18(3):125-131.

7. Tuesca R. Guallar P. Banegas JR. y Gracciani A. Factores asociados al control de la hipertensión arterial en personas mayores de 60 años en España. Revista Española de Salud Pública; 2006; 80(3):233-242.

8. Coll G. Rodríguez A. Vargas S. y cols. Ejercicio físico y calidad de vida autopercibida en el anciano hipertenso. Hipertensión y riesgo vascular; 2009; 26(5):194-200.

9. Tuesca R. La calidad de vida, su importancia y cómo medirla. Salud Uninorte; 2005; 21:76-86.

10. Pinilla A. Barrera MP. Agudelo JF. Y cols. Guía de atención de la hipertensión arterial. Guías de promoción de la salud y de prevención de enfermedades de la salud pública; 2013; 156-218.

11. Virginia M. Batista L. Lima AL. and Brandao PC. The influence of hypertension on quality of life. Arq Bras Cardiol; 2003; 100(2):164-174.

12. López PJ. Calidad de vida en paciente pediátrico con enfermedad renal crónica. Enfermería Nefrológica; 2012; 15(4):243/254. 
13. Rebollo P. Fernández F. y Ortega F. La evaluación de la calidad de vida relacionada con la salud en enfermos con hipertensión arterial. Hipertensión; 2001; 18(9):429/439.

14. Velarde E.y Ávila C.Consideraciones metodológicas para evaluar la calidad de vida. Salud pública de Mexico; 2002; 44:448-463.

15. Varela MT. Arrivillaga M. Cáceres E. Correa D. y Holguín LE. Efectos benéficos de la modificación del estilo de vida en la presión arterial y la calidad de vida en pacientes con hipertensión. Estilo de vida e hipertensión arterial; 2006; 14:69-85.

16. Gusmao JL. Mion JR. and Pierin AMG. Healthrelated quality of life and blood pressure control in hypertensive patients with and without complications; 2009; 64(6):619-629.

17. Trevisol DJ. Moreira LB. Kerkhoff A. Fuchs SC. And Fluchs FD. Health-realted quality of life and hypertension: a systemtic review and meta-analysis of observational studies. Journal of hypertension; 2011; 29(2):79-182.

18. Ninh Ha. Hoa D. Ninh Le. Vishnu K. and Moorin R. Quality of life among people living with hypertension in a rural Vietnam community. BioMed Central Public Health; 2014; 14:833.

19. Hervás A. Zabaleta A. De Miguel G. Beldarrain 0. y Díez J. Calidad de vida relacionada con la salud en pacientes con diabetes mellitus tipo 2. An Sist Navar; 2007; 30(1):45-52.

20. Grau A. Font S. Gras ME. Suñer R. Noguera J. Calidad de vida relacionada con la salud y consumo de tabaco. Intervención Psicosocial; 2007; 16(1):79-92.

21. Vilagut G. Ferrer M. Rajmil L. y cols. El cuestionario de salud SF-36 español: una década de experiencia y nuevos desarrollos. Gac Sanit; 2005; 19(2):135-50.

22. Cid J. y Damián J. Valoración de la discapacidad física: El Índice de Barthel. Revista Española de Salud Pública; 1997; 71(2).
23. Albañil T. Ramírez MC. Y Crespo R. Análisis de la calidad de vida en pacientes en hemodiálisis ambulatoria y su relación con el nivel de dependencia. Enfermería Nefrológica; 2014; 17(3):167-174.

24. Maicas C. Lázaro E. Alcalá J. Hernández P. y Rodríguez L. Etiología y fisiopatología de la hipertensión arterial esencial. Monocardio; 2003; 5(3):141-160.

25. Cavalcante M. Nogueira MT. Luna B. Camargo AC. Paola A. and Póvoa R. Quality of life of hypertensive patients treated at an outpatient clinic. Arq Bras Cardiol; 2007; 89(4):222-227.

26. Dalfó A. Badia X. Roca-Cusachs A. Aristegui I. y Roset M. Validación del cuestionario de calidad de vida en hipertensión arterial (CHAL) para su uso en España. Relación entre variables clínicas y calidad de vida. Atención Primaria; 2000; 26(2):96-103.

27. Roca-Cusachs A. Badia X. Dalfó A. y cols. Relación entre variables clínicas y terapéuticas y calidad de vida relacionada con la salud en pacientes con hipertensión arterial. Estudio Minicha. Med Clin (Barc); 2003; 121(1):12-7.

28. Barrantes-Monge M. García MJ. Gutiérrez LM. Miguel-Jaimes A. Dependencia funcional y enfermedades crónicas en ancianos mexicanos. Salud Pública de México; 2007; 49(4):459-466.

29. Ramírez-Vélez R. Agredo R. Jerez A. y Chapal L. Calidad de vida y condiciones de salud en adultos mayores no institucionalizados en Cali. Revista de Salud Pública; 2008; 10(4):529-536.

30. González J. Pariente E. Carbajo M. Tresgallo N. Ruiz E. y Peña N. Calidad de vida relacionada con la salud del anciano institucionalizado. Metas de Enfermería; 2008; 11(5):26-31. 